\title{
Orientación educativa, mercado laboral y globalización: perspectivas de empleo de los programas educativos que ofrece la Universidad Autónoma del Estado de México a los jóvenes universitarios
}

Educational orientation, labor market and globalization: employment prospects of the educational programs offered by the Autonomous

University of the State of Mexico to university students

Orientação educacional, mercado de trabalho e globalização: perspectivas de emprego dos programas educacionais oferecidos pela Universidade Autônoma do Estado do México a estudantes universitários

\author{
Rubén Gutiérrez Gómez \\ Universidad Autónoma del Estado de México, México \\ rubengut@yahoo.com.mx / rgutierrezg@uaemex.mx \\ https://orcid.org/0000-0003-1689-2317
}

\section{Resumen}

En este artículo se presenta una aproximación al estudio de las relaciones entre orientación educativa, mercado laboral y globalización. Para esto se analiza el vínculo entre el empleo y la oferta profesional de las carreras que ofrece la Universidad Autónoma del Estado de México. La intención es reflexionar teóricamente en torno a las corrientes que explican y sustentan el discurso de la orientación educativa hacia el mercado de trabajo profesional y determinar si existe una relación funcional o conflictiva que requiera generar, si es necesario, otra perspectiva de estudio e intervención de la orientación con base en la información empírica obtenida. Esto a partir de las transformaciones técnico-estructurales que ha provocado la globalización económica en el campo de trabajo.

Palabras clave: educación superior, globalización, mercado laboral, orientación educativa, orientación vocacional. 


\begin{abstract}
This article presents an approach to the study of relationship between educational guidance, labor market and globalization, to know the professional employment careers offered by the University of Mexican State. The intention of this research in addition to meet the labor demand of it educational programs offered by the institution, and to support the choice of a career of the bachelor students, is theoretically reflect the currents that explain and support the discourse of educational guidance and education to market professional work, establishing whether a functional or conflicted relationship, generating if is necessary, another perspective of analysis and intervention of guidance from the empirical information obtained. Everything from the technical and structural changes that have generated economic globalization towards the field.
\end{abstract}

Keywords: higher education, globalization, job market, educational orientation, job oriuentation.

\title{
Resumo
}

Este artigo apresenta uma abordagem ao estudo das relações entre orientação educacional, mercado de trabalho e globalização. Para isso, analisa-se a relação entre emprego e oferta profissional de carreiras oferecida pela Universidade Autônoma do Estado do México. A intenção é refletir teoricamente sobre as correntes que explicam e sustentam o discurso de orientação educacional para o mercado de trabalho profissional e determinar se existe uma relação funcional ou conflitante que exija gerar, se necessário, outra perspectiva de estudo e intervenção de a orientação baseada na informação empírica obtida. Isto é baseado nas transformações técnico-estruturais que a globalização econômica causou no campo de trabalho.

Palavras-chave: ensino superior, globalização, mercado de trabalho, orientação educacional, orientação profissional. 


\section{Introducción}

La educación superior en nuestro país ha tenido un crecimiento significativo desde finales de la década de los setenta del siglo pasado, cuando producto de las presiones sociales ejercidas durante el movimiento del 68 se empezaron a ofrecer mayores oportunidades para que las personas cursaran estudios universitarios. Desde entonces, la educación se convirtió en un mecanismo esencial para encauzar el desarrollo nacional y fomentar la movilidad social entre las clases sociales, principalmente medias y bajas.

En este sentido, los avances científicos y tecnológicos que se generaron después de la segunda conflagración mundial para afrontar la llamada Guerra Fría sirvieron para convertir a las universidades en el lugar idóneo para contribuir al mejoramiento de las condiciones de vida de una población que empezó a retomar la democracia como el modelo político predominante. En este contexto, los procesos productivos y económicos se vieron beneficiados por el desarrollo tecnocientífico, el cual fue empleado para transformar y generar los bienes y servicios que requería no solo la nueva visión del capitalismo, sino principalmente el país que buscaba satisfacer sus necesidades e impulsar su crecimiento.

Esto ocasionó que la clase media, principalmente, buscara la manera de acceder a las instituciones de educación superior (IES) — principalmente públicas - con el propósito de mejorar sus condiciones de vida y laborales. A partir de ese momento, estas casas de estudio empezaron a interesarse por evaluar cuál era el futuro de sus egresados, es decir, lo relacionado con sus posibilidades de empleo, los niveles de remuneración, los requisitos de cualificación, los sectores productivos donde se podían ubicar, etc., esto con el propósito de crear políticas de ingreso y de distribución de la matrícula cónsonas con las exigencias y la realidad del sector social y productivo.

Por este motivo, se abre la necesidad de informar a las nuevas generaciones sobre las distintas opciones educativas y ocupacionales que oferta la universidad, lo cual ha originado el surgimiento de la orientación, en principio, profesional y, posteriormente, educativa. En efecto, la orientación educativa ${ }^{1}$ nace para atender la exigencia socioeconómica de una

\footnotetext{
${ }^{1}$ La orientación educativa es un concepto que tiene un surgimiento histórico diferente. Lo vocacional — del latín vocatio, que significa 'llamado' - se refuerza en el Medioevo con la invitación a la formación religiosa en los monasterios. La industrialización generada en el siglo IX promueve el surgimiento de la orientación profesional para la ubicación laboral de la fuerza humana de trabajo. En cambio, la orientación educativa busca integrar las distintas formas de orientación en la escuela, por ejemplo, vocacional, escolar, profesional, familiar, etc. En México, en la década de los ochenta del siglo pasado, la Secretaría de Educación Pública, por mandato presidencial, establece el término orientación educativa, el cual agrupa a los distintos tipos de orientación trabajados en la educación formal.
} 


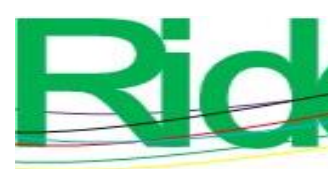

Revista Iberoamericana para la Investigación y el Desarrollo Educativo ISSN 2007 - 7467

población emergente que busca en la educación una posibilidad real para movilizarse en la estructura social y para satisfacer las insuficiencias productivas de los sectores económicos del país.

En este sentido, resulta importante estudiar y caracterizar la relación entre universidad, mercado laboral y orientación con el fin de identificar sus propiedades y generar información más acorde con la realidad estructural de mercado para determinar las posibilidades que este ofrece a las distintas carreras profesionales.

A partir de lo anterior, se tiene que formar - y no solo informar - al estudiante sobre las variables que debe considerar cuando elige una carrera que formará parte de su proyecto de vida. Para esto, sin embargo, se debe reflexionar primero en torno a la tarea que debe cumplir la orientación educativa (OE) en el campo vocacional para incorporar otras perspectivas teórico-metodológicas que permitan ofrecer al estudiante una visión más amplia de las posibilidades de empleo que tiene cada una de las carreras universitarias.

Ahora bien, es cierto que esta información se ha venido proporcionando a través de instrumentos profesiográficos, como catálogos de carreras, exporientas, folletos y conferencias profesiográficas. No obstante, estos solo se usan para recabar información, y no para problematizar la compleja relación que existe entre educación y mercado en este contexto de globalización económica, lo cual permitiría conformar un esquema teórico, conceptual y metodológico no solo para comprenderla y explicarla, sino esencialmente para intervenir en el proceso de elección de carrera del estudiante de bachillerato.

Por esta razón, en este documento se presenta una aproximación al estudio de esa relación para tratar de fundamentar la manera en que ellas se articulan como prácticas socioacadémicas y económicas en un contexto de crisis como el que se vive en la actualidad.

\section{Problemática de la relación entre orientación, mercado de trabajo y educación}

Desde principios de la década de los ochenta del siglo pasado se ha reconocido en documentos oficiales que una de las causas del crecimiento de la matrícula en la educación superior - especialmente a nivel de pregrado - se encuentra en la falta de información sistemática sobre las distintas opciones tanto educativas como laborales que se ofrecen en cada una de las entidades federativas (Hernández Laos, 2012). Esto ha ocasionado que se haya sugerido reforzar los servicios de orientación en cada una de las instituciones educativas para que el estudiante, al concluir sus estudios y previo a su elección profesional, pueda contar con 


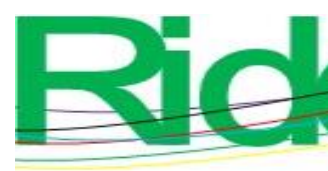

Revista Iberoamericana para la Investigación y el Desarrollo Educativo ISSN 2007 - 7467

los argumentos esenciales para decidir de la forma más adecuada posible la carrera que desarrollará profesionalmente.

Los resultados de este exhorto, sin embargo, parecen no ser los indicados, pues este crecimiento sin precedentes ha provocado la concentración de la matrícula en unas pocas carreras donde se concentra más de la mitad de estudiantes, lo cual ha generado una sobreoferta de mano de obra calificada que intenta incorporarse en un mercado laboral que ha sido incapaz de absorber a todos los egresados de la educación no solo del nivel superior, sino incluso de los niveles medios terminales. Aunado a esto, muchas de las opciones de nivel superior que se han ampliado en las IES son carreras con baja demanda o poco reconocimiento en el mercado laboral, por lo que el egresado se ve en la necesidad de ir abriendo espacios laborales para campos disciplinares poco conocidos. En otras palabras, la proporción de alumnos que demandan, ingresan y concluyen sus estudios del nivel superior, principalmente en carreras con mayores índices de matrícula, no se corresponde con las opciones de empleo que hay en el mercado.

$\mathrm{Al}$ respecto, distintos estudios indican que solo uno de cada diez jóvenes que ingresan al sector productivo lo hacen en condiciones estables (Gandini, 2004). Más aún, esos reportes señalan la brecha que se ha venido ampliando entre la demanda de ingreso a la institución y la demanda de profesionales por parte del mercado laboral. Esta problemática ha servido para concretar distintas investigaciones que intentan explicar y determinar las causas del desempleo y subempleo profesional. En ese sentido, los estudios realizados han encontrado que el vínculo educación-empleo va más allá de una simple relación funcional, ya que el mercado laboral presenta una estructura heterogénea y segmentada que impone ciertos mecanismos de inserción que la mayoría de las veces queda fuera del alcance de los egresados (Magaña, 1992; Muñoz y Hernández Garibay, 1992).

Como se mencionó anteriormente, no se pueden dejar de lado las transformaciones del capitalismo a nivel mundial por la globalización económica, la cual ha propiciado de forma significativa la contracción del empleo debido a las dificultades del sector productivo por "ubicar" sus productos en un mercado en el que los niveles de ingreso de las clases trabajadoras se han devaluado y apenas permiten acceder a la canasta básica familiar.

Asimismo, los efectos de la globalización económica y el desarrollo de las tecnologías de la información y comunicación (TIC) han estado a la par de una recomposición de los mercados en los que la producción de bienes y servicios se ha fragmentado en distintos países 
y las empresas tienden a fusionarse para obtener mejores rendimientos económicos. En efecto, el desarrollo de la cuarta revolución industrial (Castells, 1994) ha venido acompañada de un incremento en la mecanización y robotización de la producción del trabajo, lo que ha desplazado a la mano de obra y ha obligado a los empresarios a modificar sus criterios de selección de personal, entre los cuales sobresale la escolaridad, pues es uno de los procesos que permite la obtención de competencias para formar el capital humano requerido por los nuevos procesos productivos (Muñoz Varela, 2012).

Esta situación se ha visto expresada en los cambios suscitados en la economía mundial, cuya tendencia hacia el globalismo económico ha traído como consecuencia ajustes y políticas tendientes a conformar bloques económicos regionales que permitan la integración de distintos países con mejores condiciones arancelarias para poder comerciar con menos barreras (Dettmer, 2004). No obstante, la tendencia de potencias como Inglaterra y Estados Unidos de modificar su política económica no se corresponde con esa iniciativa, pues se han abandonado o modificado las relaciones con los bloques que esos mismos países promovieron hace tan solo algunas décadas.

Para el caso de nuestro país, la firma del Tratado de Libre Comercio con los países vecinos del norte dejó de manifiesto la política neoliberal que dio la pauta al globalismo de la región, lo cual se constituyó en la punta de lanza para establecer un mercado común latinoamericano que, sin embargo, con la llegada de Donald Trump al poder ha propiciado ajustes en las políticas y en la normatividad establecida.

A pesar de ello, nuestro país ha continuado con la política de apertura y el otorgamiento de concesiones para que los capitales extranjeros inviertan en nuestro territorio, como sucedió con la reciente reforma energética llevada a cabo en la actual administración federal. Estas y otras acciones, como la participación de capital financiero trasnacional en operaciones bancarias o la privatización de las empresas estatales y paraestatales, representan la entrada a un contexto más competitivo en el que la estructura laboral del país modificará las condiciones y requisitos de acceso al mercado de trabajo, entre las que destacan las de índole educativo.

De esa forma, la educación mexicana — especialmente la de nivel superior — tiene ante sí un reto para formar la mano de obra calificada que enfrentará las nuevas vicisitudes que se esperan en esta década, en un mercado cada vez más competitivo y restringido en el que el desarrollo científico y tecnológico exige nuevas competencias a nivel internacional (Muñoz Varela, 2012). Por ende, es importante conocer la dinámica del empleo para intentar 


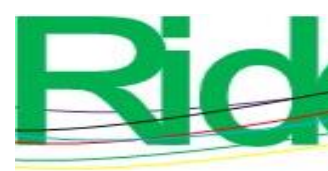

Revista Iberoamericana para la Investigación y el Desarrollo Educativo ISSN 2007 - 7467

pronosticar las posibilidades que tienen los egresados de acceder al mercado laboral, ya que la apertura económica se ha convertido en una política de largo alcance cuyos resultados inmediatos se deben examinar permanentemente (Dettmer, 2004).

Eso quiere decir que la tarea de la $\mathrm{OE}$, en su área vocacional-profesional, debe centrarse en estudiar este fenómeno para informar y formar reflexivamente a los estudiantes de educación media y media superior en torno a su perfil vocacional y su plan de vida y de carrera (Alles, 2005; Castañeda, 2014). Para ello, se debe no solo concienciarlos sobre la supuesta relación entre educación superior y ascenso socioeconómico, sino también comprometerlos en la búsqueda de datos objetivos que les permitan conocer los distintos sectores económicos y los criterios de inserción laboral.

Esto, por supuesto, también implica trabajar en la formación del orientador educativo, ya que este profesional es el responsable de explicar a los estudiantes de bachillerato las distintas opciones educativas del nivel superior, así como las posibilidades de empleo profesional en el contexto estatal, nacional e internacional. Con esta tarea se busca romper los esquemas anquilosados en la práctica orientadora y en el propio ejercicio del orientador. Es decir, resulta fundamental que este especialista domine otras perspectivas teóricometodológicas que le permitan revitalizar su práctica para convertirse en un sujeto de apoyo y transformación del plan de vida y carrera del estudiante. En definitiva, el papel de la OE no se debe quedar solo en el acopio de información mediante tests psicométricos para describir el perfil vocacional y profesional del estudiante, sino que debe procurar evaluar la realidad para identificar los factores que la determinan.

\section{Teorías que explican la relación entre orientación, educación, mercado laboral y globalización}

\section{Paradigma neoclásico}

Los primeros trabajos que empezaron a reconocer a la educación como un elemento estrechamente vinculado con la producción fueron realizados en los años cincuenta del siglo pasado. De estos sobresale el estudio de Theodore Schultz, quien analizó la relación entre el gasto en educación y la formación en capital físico en Estados Unidos durante el periodo 19001956. En su trabajo este investigador demostró que la inversión en educación resultaba 3.5 veces más atractiva que la inversión en capital físico (Carnoy, 1988). A partir de entonces 


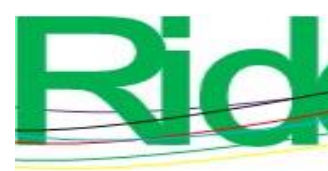

Revista Iberoamericana para la

surge la teoría del capital humano $(\mathrm{TCH})$ como un enfoque teórico inicial para el estudio de las relaciones entre orientación, educación y mercado de trabajo.

Denison, por su parte, al considerar los factores que influían en la tasa de crecimiento del producto interno bruto (PIB) de Estados Unidos, detectó que existía un factor residual que se vinculaba con una mayor productividad de la fuerza de trabajo, lo cual era el resultado de una mejor educación. El capital humano, por consiguiente, empezó a ser considerado como una variable que hasta la fecha no se había tomado en cuenta en la producción (Navarro, 2014; Pescador, 1985).

Ahora bien, la TCH lleva implícita una decisión "individual” para obtener niveles educativos elevados, los cuales se sustentan en una elección vocacional que busca acoplar las característica personales del individuo con las de la profesión que pretende estudiar. Esto sugiere, en principio, que los individuos "gozan de una libre elección al maximizar su utilidad bajo ciertas restricciones [y que] su conducta está configurada como si estuviesen adquiriendo educación en forma de inversión de capital humano que les permitirá mejorar al máximo sus ingresos por el resto de su vida" (Pescador, 1985). No es casual, por tanto, que la orientación profesional apareciera formal y sistemáticamente a principios del siglo pasado en Norteamérica con la obra de Frank Parsons Choosing a Vocation, es decir, en pleno auge del capitalismo a nivel mundial, lo cual sirvió para crear una oficina orientadora que funcionó desde un punto de vista empírico-práctico (Mira, 1980).

En este sentido, las implicaciones que ha tenido TCH no solo se han encaminado a la educación-ingresos, sino que han abarcado otras dimensiones, como el papel del progreso científico y tecnológico en la educación y en el trabajo. Por ello, se considera que el papel principal del sistema educativo es servir de mecanismo social en la acumulación y trasmisión de conocimiento científico-técnico funcional para las necesidades de la producción.

En efecto, el progreso científico ha incrementado el nivel de calificación de la fuerza de trabajo en virtud de la tendencia por disminuir el trabajo operativo y repetitivo como consecuencia del proceso de automatización. A medida que aumentan los requisitos de calificación para todas las ocupaciones debido al continuo progreso científico y técnico, se vuelve cada vez más necesaria la articulación entre formación y ocupación, lo cual refuerza el postulado de que el papel del sistema educativo es cumplir eficazmente una función técnica en la producción. En otras palabras, otorgar el conocimiento requerido por cada ocupación 


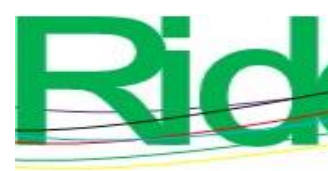

Revista Iberoamericana para la Investigación y el Desarrollo Educativo ISSN 2007 - 7467

según sea definida en la división del trabajo, así como asociar el empleo y la productividad de la fuerza laboral al tipo y nivel de acreditación educativa adquirida.

En tal sentido, el enfoque neoclásico establece que el subdesarrollo económico es en gran medida explicado por el subdesarrollo educativo, el cual es definido en función del grado de imitación de los parámetros de desarrollo educativo en los países industrializados. Como puede observarse, este enfoque se fortalece en el proceso de globalización económica en países emergentes como el nuestro. Asimismo, explica el crecimiento de la demanda educativa como consecuencia de los postulados de crecimiento económico y distribución del ingreso en una población hasta entonces muy limitada en sus expectativas laborales y económicas.

\section{La orientación y el paradigma neoclásico}

Los postulados anteriores son asumidos por la OE en tanto busca ajustar el perfil personal (intereses y aptitudes) a las características de una profesión (Gutiérrez, 1999). Esta visión se asume como una relación lineal, funcional y adaptativa que omite la complejidad estructural de la educación superior y el mercado de trabajo. De esta manera se ubica como el enfoque actuarial, psicologista y profesiocéntrico (Bohoslavsky, 2000; Nava, 1993; Osipow, 1996) en el que solo las características personales deben concordar con las de la profesión elegida. En palabras de Taylor, solo se debe buscar "al hombre adecuado en el puesto adecuado" (citado por Prawda, 2002), pues de esa manera se puede garantizar al mercado el suministro del capital humano requerido por la producción material de la sociedad (teoría adecuacionista).

Desde esta concepción, en los años sesenta del siglo pasado, se plantea la necesidad de que la orientación vocacional informe al estudiante de nivel medio y medio superior sobre el abanico de oportunidades profesionales que existe en las universidades, de modo que no se remita exclusivamente a las carreras que en la posguerra despuntaron en la modernización y en "desarrollo" socioeconómico del país, como como Derecho, Administración, Contaduría, Medicina, Arquitectura, etc. En este sentido, la Asociación Nacional de Universidades e Instituciones de Educación Superior (ANUIES) es la institución que empezó a fomentar —a finales de la década de los setenta del siglo pasado- el desarrollo de la orientación no solo planteando la necesidad de crear y fortalecer los servicios de orientación vocacional en cada uno de los planteles institucionales, sino también generando instrumentos profesiográficos (catálogos de carreras, carteles, folletos, exposiciones gráficas, videos, conferencias y test psicométrico-vocacionales) para apoyar la elección de carrera del estudiante. Esta iniciativa, 


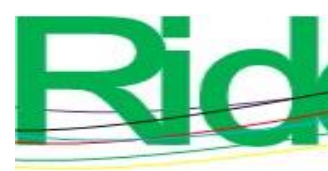

Revista Iberoamericana para la Investigación y el Desarrollo Educativo ISSN 2007 - 7467

sin embargo, no ha conseguido los resultados esperados, pues en la actualidad las mencionadas carreras siguen siendo las que agrupan a un mayor porcentaje de la matrícula.

Efectivamente, en la Universidad Autónoma del Estado de México (UAEM) esta realidad se ha constatado en estudios sobre la demanda de las carreras de nivel superior, los cuales han demostrado que más de $50 \%$ de la matrícula cursa solo diez carreras, entre las que sobresalen Contador Público, Administración, Derecho, Médico Cirujano y Arquitectura (Gutiérrez, 2015). Más aún, la razón principal por la que los aspirantes prefieren estudiar estas carreras se relaciona con la obtención de un empleo seguro y altos ingresos, lo cual les permitiría mantener o movilizar su estatus socioeconómico. Es decir, en el imaginario social del estudiante de bachillerato la inversión educativa garantiza el ingreso al mercado laboral.

Como se observa, desde los postulados del paradigma neoclásico, el problema de la elección de carrera se reduce a un asunto informativo, en el que la orientación debe ser la encargada de generar y proporcionar datos profesiográficos y psicométricos para que el estudiante conozca sus características personales, así como la oferta educativa de nivel superior, con el propósito de concretar una elección "racional" que se ajuste a los propósitos individuales y sociales. Esto significa que la orientación estaría encaminada a apoyar la distribución racional de la fuerza de trabajo profesional para atender las necesidades del sector productivo y, en consecuencia, promover el desarrollo en un contexto en el que aparentemente el sector productivo funciona equilibrada y equitativamente para todos (Cortada, 1980).

Esta visión orientadora se inscribe en el modelo desarrollista funcional planteado por Bilbao (1986), pues busca adaptar al futuro profesionista a las necesidades que requiere el desarrollo social, político y económico del país. Para esto le proporciona información no solo sobre de sus característica personales, sino también sobre las distintas opciones de educación superior para que llegue a una elección "racional" de la carrera (Nava, 1993).

\section{Paradigma crítico}

Contrario a la visión neoclásica, el paradigma crítico establece que las relaciones entre la educación y el sistema productivo son el resultado de un largo proceso histórico y sistémico de lucha, contradicción y conflicto entre intereses de grupos y clases sociales antagónicas, en donde las "decisiones libres" de los individuos reflejan las decisiones de esas clases sociales. En este paradigma el sistema productivo se determina por vínculos que definen qué, cómo y para quién se distribuye socialmente la producción, de ahí que sea la naturaleza de esas 


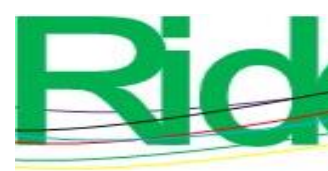

Revista Iberoamericana para la

relaciones sociales las que establezcan las opciones técnicas y organizacionales en la producción (Gómez, 1982). Al respecto, Carnoy (1988) señala lo siguiente:

Los aumentos de la instrucción escolar no han conducido a tasas más elevadas de desarrollo, sino más bien al reemplazo de la mano de obra menos instruida por otra más instruida y al incremento de la instrucción escolar promedio entre los desempleados, puesto que el número de trabajos no ha aumentado tan rápidamente como el de personas instruidas.

En el sistema capitalista, las relaciones de producción más relevantes para entender la articulación entre el sistema productivo y el educativo establecen que la mayoría de la población no posee bienes de producción, por lo que se ve obligada a vender su fuerza de trabajo a los dueños de estos. En tal sentido, el mercado de trabajo es la institución necesaria para la compra-venta de una fuerza laboral heterogénea y para su distribución en las diferentes ocupaciones y oficios. En consecuencia, la acreditación educativa desempeña cada vez más un papel predominante para la selección y exclusión de acceso al trabajo.

Así, las decisiones en la producción son disposiciones cuya especificidad técnica depende de aspectos sociopolíticos derivados de la naturaleza de las relaciones sociales de producción dominantes. Esto significa que aun cuando la escuela es la institución que provee las calificaciones de la fuerza laboral, la selección de esta está determinada por mecanismos exógenos a lo educativo, tales como el nivel socioeconómico del aspirante, la oferta de trabajo, las prácticas de selección de cada empresa, las normas organizacionales de la empresa, los segmentos ocupacionales, la discriminación sexual y racial, etc. Por eso, se puede afirmar que no es el sistema educativo el que establece el volumen global del empleo en la economía, y tampoco son las particularidades educativas las que explican las desigualdades económicas. La educación, en consecuencia, solo es una variable que puede aumentar la probabilidad de acceso a ciertas ocupaciones, pero esto no significa que garantice el empleo ni la calidad de este (Ruiz del Castillo, 2001).

Ante esta realidad cabe cuestionarse, entonces, sobre cuál es el papel que cumplen las instituciones educativas. En palabras de Carnoy (1988), la escuela se convierte en la instancia de "elaboración y difusión de valores, actitudes y conductas congruentes con las relaciones sociales de producción que sirven para fortalecer y legitimar la estructura social existente". Esto quiere decir que las instituciones educativas no son únicamente lugares para crear 


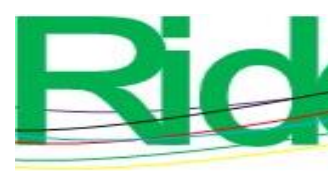

Revista Iberoamericana para la Investigación y el Desarrollo Educativo ISSN 2007 - 7467

destrezas vocacionales, sino también espacios para trasmitir cultura y valores que puedan canalizar a los educandos hacia diversos papeles sociales en pro del orden social establecido.

Lo anterior ha servido para considerar a la educación y al mercado de trabajo como una relación conflictiva y contradictoria que en una sociedad de libre mercado difícilmente encuentra una adecuación óptima entre oferta y demanda laboral. En estas circunstancias, el mercado de trabajo profesional representa a la institución destinada a la compra-venta de una fuerza laboral heterogénea que la distribuye en las diferentes ocupaciones y oficios determinados por las características intraorganizacionales e interorganizacionales de las empresas del sector productivo de un país. Esto ha llevado a ubicar al mercado como un espacio diferencial que aparece estructuralmente dividido en varios mercados desiguales y segmentados que conforman los grandes niveles jerárquicos intraorganizacionales: concepción y gestión de la producción (intelectual), técnico-administrativo, y de ejecución de la producción (trabajo manual), así como las diferencias salariales y condiciones de trabajo que dependen de las características económicas de cada empresa, tales como intensidad del capital, complejidad técnica, tamaño de la empresa, etc. (Gómez, 1982).

Así, surgen algunas propuestas alternativas, como la teoría dualista y la teoría de la segmentación del mercado de trabajo (Escotet, 2002; Pescador, 1985). La primera plantea que el mercado se divide en dos sectores: el primario (caracterizado por mejores empleos, altos salarios, condiciones de trabajo satisfactorias, estabilidad y amplias posibilidades de promoción), y el secundario, en el que se ubican los empleos menos atractivos y los salarios bajos con condiciones de trabajo menos satisfactorias; este sector lo ocupan las personas que provienen de las clases sociales bajas, las mujeres y quienes tienen menor educación, hábitos incorrectos de trabajo y menor productividad.

La teoría de la segmentación, por otra parte, plantea que la estructura ocupacional se divide en fragmentos cualitativamente diferentes, persistentes y estables, con poca movilidad entre ellos, y cada uno corresponde a divisiones sociales, sexuales y económicas preexistentes en la sociedad, las cuales son el resultado del proceso histórico del desarrollo capitalista que se basa en el control de la fuerza de trabajo y del proceso productivo (Navarro, 2014; Márquez, 2011). 


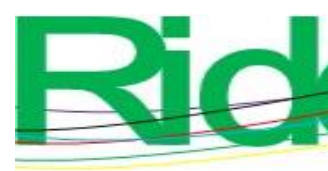

Revista Iberoamericana para la Investigación y el Desarrollo Educativo ISSN 2007 - 7467

Con se puede observar, para acercarse al estudio del vínculo educación-mercado se debe partir de una perspectiva teórica que permita comprender la naturaleza de dicha relación.

\section{La orientación y el paradigma crítico}

Las reflexiones y los cuestionamientos planteados a la práctica psicométrica y profesiocéntrica de la orientación educativa - como consecuencia de la poca movilidad de la matrícula de educación superior, así como del aplazamiento de soluciones a la problemática psicosocial, pedagógica y de salud de los jóvenes estudiantes del nivel medio superior — han permitido readecuar sus fundamentos, métodos y prácticas institucionales.

Para el caso del objeto abordado en este documento - es decir, la relación entre orientación, educación y mercado de trabajo en la globalización-, la orientación asume los planteamientos del enfoque crítico a la luz de la teoría sociopolítica, la cual plantea que no existe un equilibrio entre la oferta y la demanda de egresados de la educación superior porque es el mercado de trabajo el que determina la cantidad y los requerimientos de la fuerza laboral calificada.

Aunado a esto, el sector productivo no ha sido capaz de crear los puestos necesarios para absorber al total de los egresados de la educación superior debido a las condiciones estructurales de la economía que prevalece en los países emergentes y los cambios que ha traído la revolución tecnológica en el marco de la globalización.

En este sentido, la problemática de la elección de carrera para la OE transciende el aspecto informativo, ya que elegir una carrera profesional implica un proceso de reflexión y análisis de las distintas condiciones económicas, políticas, sociales y educativas del contexto en el que se toma la decisión. Estos planteamientos críticos de la elección vocacional se sustentan en el paradigma del desarrollismo crítico de la orientación. En este se cuestionan las condiciones históricas, sociales y políticas en las que la OE tuvo un auge importante para el apoyo en la redistribución de la matrícula hacia áreas científicas y productivas necesarias para el desarrollo económico del país (como el sector agropecuario, la industria de la transformación y de las ciencias básicas), sin tomar en cuenta que el progreso es un problema estructural de los países emergentes mantenido históricamente por las metrópolis en la fase más alta del capitalismo (Bilbao, 1983; Nava, 1993). 


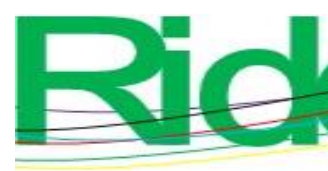

Revista Iberoamericana para la Investigación y el Desarrollo Educativo ISSN 2007 - 7467

Efectivamente, las clases sociales medias, al considerar a la educación como un canal de movilidad social, únicamente han basado su elección de carrera en los estereotipos vocacionales generados por los medios de comunicación masiva (cine, televisión, revistas), los cuales han servido para construir un imaginario social de ascenso laboral y económico promovido por el estudio de determinadas carreras.

Ante ello, la $\mathrm{OE}$ busca distintas estrategias teórico-metodológicas que permitan al sujeto identificar no solo el amplio abanico profesional que ofrece la educación superior, sino también las condiciones sociohistóricas en las que se gesta este proyecto de desarrollo, analizando la conformación de su trayectoria vocacional a partir de sus intereses personales, familiares, culturales, sociales y económicos, desideologizando y contextualizando con esto la elección de carrera.

\section{Paradigma integracionista: neoliberal-globalizador}

Las transformaciones estructurales generadas por la globalización y por el desarrollo tecnológico en la dimensión de los sistemas económico-productivos, las estructuras del empleo y los perfiles profesionales se caracterizan por los requerimientos de la "apertura global" de la economía y por una mayor incorporación de las TIC en múltiples procesos de carácter básico no solo en la economía, sino en todas las demás actividades que las personas llevan a cabo en la sociedad (Muñoz Varela, 2012).

Se insiste, por tanto, en que la formación debe estar basada en el desarrollo de habilidades cognitivas y operacionales esenciales para ser competitivo, innovador, flexible, eficiente y eficaz. Estas son las nuevas demandas que establece el mercado de trabajo en el contexto de la globalización económica y de la sociedad de la información y el conocimiento. En este contexto del proceso globalizador, el cual se consolida en el presente con la era de la llamada sociedad del conocimiento, surge el enfoque por competencias, el cual viene a establecer nuevas vertientes para estudiar la relación entre orientación educativa, mercado laboral y globalización (OEMG).

Cobijadas por las políticas globales de corte pragmatistas, tecnologistas y de reconversión industrial basada en el conocimiento, las IES se integran a un proceso "modernizador" que tiene a redefinir las cualificaciones y competencias que deben adquirir los futuros egresados para insertarse en un mercado laboral cada vez más competitivo, aunque 


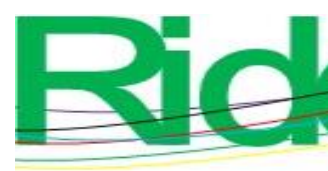

Revista Iberoamericana para la Investigación y el Desarrollo Educativo ISSN 2007 - 7467

también deprimido, especialmente en la industria manufacturera de alta tecnología y de servicios comerciales, financieros y sociales (Didriksson, 1997; Valenti y Castillo, 1997). En este sentido, la concepción de competencia que aparece en la década de los noventa del siglo pasado se ha venido implementando paulatinamente en instituciones educativas de varios países (entre ellos México) para intentar formar a personas con ciertas habilidades, conocimientos y actitudes que les posibiliten un mayor impacto en su inserción laboral. Al respecto, Tobón (2006) considera lo siguiente:

En la consolidación del enfoque de competencias en la educación (...) ha influido el momento histórico y la economía. Y eso se debe tener en cuenta para tener un criterio crítico en su empleo. En lo social, se tienen las crecientes presiones para que la educación forme para la vida y para el trabajo con calidad, y trascienda el énfasis en lo teórico y la mera transmisión de la información, pues con la paulatina emergencia de la Sociedad del Conocimiento (sic), lo más importante no es tener conocimientos sino saberlos buscar, procesar, analizar y aplicar con idoneidad (...). Con respecto a lo económico, ha crecido la demanda de las empresas a las instituciones educativas para que formen profesionales idóneos, de tal manera que esto les permita competir con otras empresas nacionales e internacionales para mantenerse y crecer (...). En síntesis, el auge de las competencias en la educación se corresponde con una mayor implicación de la sociedad en la educación, la cultura de la calidad, la globalización y la competitividad empresarial (p. 4).

Esto ha llevado a plantear nuevos elementos para comprender y explicar la relación OEMG:

Vivimos en una época en la cual la información aplicada a las esferas de la producción, de la distribución y de la gestión está revolucionando las condiciones de la economía, el comercio, las bases de la política, la comunicación cultural mundial y la forma de vida y de consumo de las personas. Este nuevo ciclo ha sido denominado sociedad de la información, debido a que es la información la que ahora dirige la economía global que está surgiendo. El incremento y la complejidad de los problemas que este giro promueve es quizá más perceptible en la educación superior, debido al cambio 


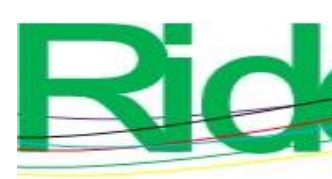

Revista lberoamericana para la Investigación y el Desarrollo Educativo ISSN 2007 - 7467

de las estructuras sociales, entre las cuales la globalización (como un factor externo) ha sido un aspecto importante y por el hecho de que la educación superior por sí misma se ha visto obligada a proponer cambios internos radicales como resultado de la situación explosiva en el incremento de alumnos, profesores y administrativos. Además, porque al ser el alumno egresado quien se enfrenta precisamente a los nuevos retos de la oferta y la demanda, encara grandes problemas, tales como elegir, analizar y emplear la información, investigar y generar procesos y técnicas innovando los existentes, que hacen evidente la necesidad de un aprendizaje distinto y permanente. La educación superior necesita ahora una visión renovada para su planeación, que sea congruente con las características de la sociedad de la información, como por ejemplo, el desarrollo y promoción de las nuevas tecnologías, las cuales en la actualidad amplían las fronteras y transfiguran ya el proceso de enseñanzaaprendizaje. Por ello se ha visto que es necesario repensar los conceptos básicos de la planeación estratégica de las universidades y explorar las competencias que las instituciones de educación superior forzosamente requerirán para poder anticipar las exigencias a las que sus alumnos se enfrentarán en el siglo XXI (Argudín, 2015, p. 1).

Las implicaciones científico-técnicas son consideradas en los procesos productivos que se desarrollan para la consolidación de la globalización económica, lo cual genera otros enfoques teóricos de investigaciones más recientes, tales como la teoría adecuacionista, teoría de la sobreeducación y teoría de la movilidad profesional (Navarro, 2014). La primera señala que cada nivel y especialidad en el trabajo se relaciona con un conjunto de categorías laborales (Salas, 2009, citado en Navarro, 2014). Esa relación constituye la forma más óptima de intercambios en el mercado de trabajo, por lo que resulta esencial "adecuar" las necesidades productivas con los conocimientos y habilidades que desarrolla la escuela en los estudiantes durante su formación académica. En la globalización económica este planteamiento se ha reforzado porque el modelo neoliberal de la educación plantea una relación más estrecha con el sistema productivo, pasando de un modelo enciclopedista a uno evaluador (Mendoza, 1997). Por eso, las IES tienden a estrechar los vínculos de las competencias laborales con las empresas que contratan a sus egresados. 


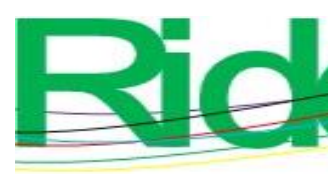

Revista Iberoamericana para la Investigación y el Desarrollo Educativo ISSN 2007 - 7467

La teoría de la sobreeducación, en cambio, intenta medir el desajuste entre la cualificación de los individuos y la inherente a los puestos de trabajo, bajo el supuesto de que a cada trabajo "le corresponde un determinado tipo de habilidades (...), pudiéndose determinar el grado de ajuste o desajuste de las personas en función del tipo de formación adquirida y de las características del trabajo que desempeñan" (Navarro, 2014). De esta manera se determina si las funciones y cualificaciones establecidas en el perfil del puesto de trabajo se ajustan a la formación del profesionista requerido. Si no hay correspondencia entre el perfil y la formación adquirida, o si hay un desfase entre los conocimientos y las habilidades del puesto en relación con la formación de quien desempeña el trabajo, se determina si existe una sobreeducación laboral. Estos planteamientos se han visto complementados con la teoría del acoplamiento al puesto de trabajo.

Por otra parte, la teoría de la movilidad profesional señala que existe un cambio laboral permanente en las empresas debido a las cualificaciones superiores de los trabajadores, lo cual se evidencia tanto de forma interna (promociones salariales y de puestos de trabajo) como de forma externa (cambio de empresa o ámbito laboral). Desde esta perspectiva, es recomendable adquirir conocimientos y experiencias para el desempeño en el trabajo (sea en la misma empresa o en otra), pues esas variables resultan significativas para alcanzar mejores niveles salariales y laborales.

Otras perspectivas generadas recientemente señalan que el individuo adquiere su formación a partir de diversas experiencias y recursos a lo largo de la vida, de ahí que se considere que la educación formal no es la única promotora de aprendizajes, habilidades y actitudes para el desempeño de una actividad productiva. Esto quiere decir que existe una diversificación en las trayectorias formativas (Planas, 2003). Por eso, el desarrollo de las competencias debe sustentarse en el estímulo de múltiples experiencias académicas, sociales y culturales que se deben promover dentro y fuera del contexto escolar para que el estudiante reconozca metacognitivamente cómo se pueden afianzar sus habilidades cognitivas, operacionales y actitudinales en pro de su incorporación al mercado de trabajo.

Por último, se halla el planteamiento de la expansión educativa, la cual indica que la oferta y la demanda educativa mantienen cierta autonomía respecto a la dinámica del mercado laboral. En esta teoría se reconocen propósitos distintos entre la educación y el mundo del trabajo, con lógicas de desarrollo diferentes entre sí (Navarro, 2014). Este planteamiento intenta explicar la brecha que se abre entre educación y mercado. 


\section{La orientación y la perspectiva integracionista}

La OE - como se concibe en este ensayo - debe considerar la perspectiva sociopolítica para superar y cuestionar la visión profesiográfica y testista, pues de esa manera se puede pasar a una óptica de conflicto, donde la relación entre orientación y mercado de trabajo obliguen a reflexionar sobre la problemática de la elección como un vínculo complejo y conflictivo que debe superar su carácter ideológico, principalmente ante los retos a los que la someten las transformaciones estructurales de la globalización.

Se requiere, por ende, un enfoque integracionista que permita "depsicologizar" a la OE para llegar a una visión multidisciplinaria y transdisciplinaria (que incluya a la pedagogía, la sociología, la historia, la antropología y la política) con el fin de explicar y abordar el proceso de elección vocacional del estudiante (Nava y Muñoz, 1990). Desde esta perspectiva, se reconoce la multiplicidad de variables y procesos históricos, sociales, culturales, económicos y pedagógicos que entran en juego en la elección de carrera, por lo que resulta pertinente tomar en cuenta los factores personales y medioambientales que influyen en las preferencias de los sujetos (Nava, 1993; Rascovan, 2014).

Lo anterior deja en claro que si el surgimiento de la OE en América se produce a partir de la necesidad de apoyar al ciudadano a elegir la carrera u ocupación que mejor se ajuste a sus intereses, habilidades y potencialidades personales, entonces lo vocacional, lo ocupacional y la carrera se convertirán en el eje que sustentará la labor de la orientación, en donde sus distintas áreas de intervención confluirán en la construcción del proyecto vocacionalprofesional (Bisquerra, 1998; Mira, 1980), como se aprecia en la figura 1. 
Figura 1. Áreas de confluencia de la orientación educativa en el marco de la globalización

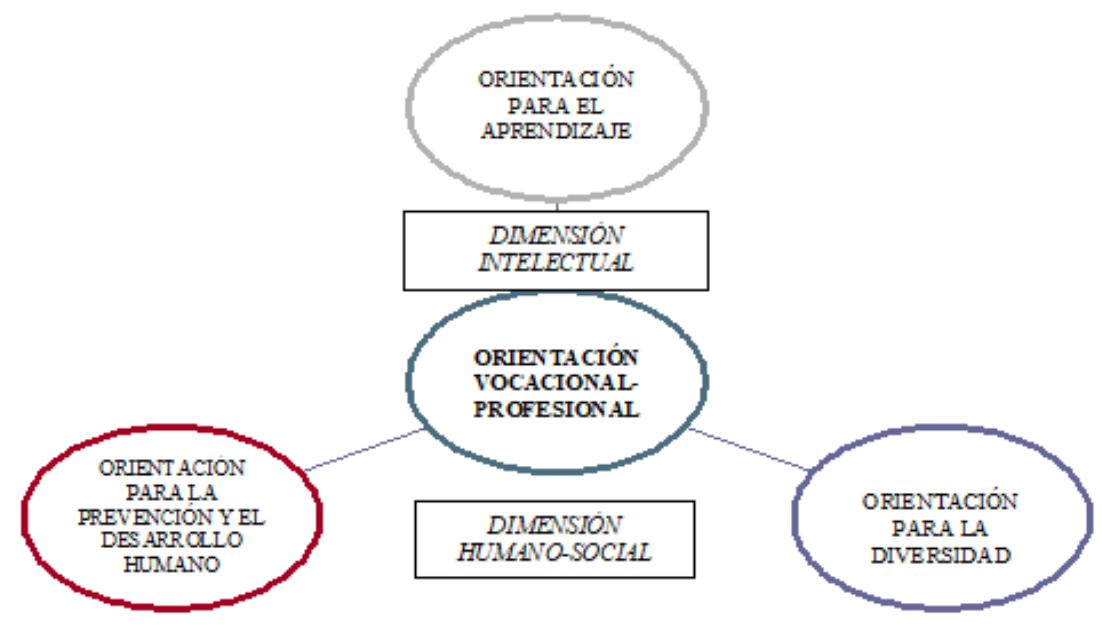

Fuente: Elaboración propia

En la figura 1 se puede apreciar que lo vocacional está integrado por la "orientación para el aprendizaje y la permanencia escolar", las cuales forma parte de la dimensión intelectual, pues el desarrollo de estrategias para el aprendizaje apoya la permanencia en la escuela y, por tanto, la continuidad hacia estudios de nivel superior. Asimismo, se articula con la orientación para la prevención, el desarrollo humano y la diversidad, correspondientes a la dimensión humano-social. Esta se refiere a los elementos asociados al desarrollo humanosocial del estudiante (considerado como "ciudadano del mundo" en el marco de la globalización), lo cual le permite reconocer, asumir y respetar la diversidad presente en las relaciones educativas, sociales y culturales en las que se desenvuelve.

Con esto se muestra la complejidad que debe asumir lo vocacional-profesional como práctica educativa, ya que debe tomar en cuenta la pluralidad de elementos que constituyen el campo de la orientación. Esto significa que la $\mathrm{OE}$ no debe ser concebida como un área disciplinaria simple de abordar, pues no se limita solo a aplicar instrumentos psicológicos, vocacionales e informativos para enseñar las alternativas de estudio, sino que supone un proceso complejo de apoyo para la construcción vocacional del sujeto, cuyo desempeño socioprofesional impacta en la definición y conducción de políticas y propósitos de desarrollo nacional y mundial.

Aunado a esto, se debe subrayar que si bien es cierto que la práctica de la orientación también ha asumido el enfoque por competencias como un mecanismo de apoyo para la permanencia, el desarrollo (académico, personal, vocacional) y la conclusión de los estudios 
de nivel medio superior (Subsecretaría de Educación Media Superior de la SEP, 2008), también es cierto que aún queda pendiente evaluar la forma en que se ha incorporado este enfoque a la OE, y principalmente su impacto en la conformación de su PVyC vinculado a la elección de carrera y a su desarrollo profesional en el mercado laboral actual (Tovar, 2011).

Sin duda alguna, el proceso globalizador de la economía ha complejizado la dinámica y la estructura del mercado de trabajo profesional, por lo que la orientación debe integrar en su ser y hacer una perspectiva que reconozca la diversidad de elementos que condicionan la elección de una carrera profesional y el efecto que esta tendrá en la vida posterior. Como indica Rascovan (2014), desde una visión sociopolítica, lo vocacional se asume como la forma de elucidar los fenómenos sociohistóricos que la determinan, develando las lógicas, ideologías e intereses que mueven las pugnas entre educación y mercado de trabajo. Por tal motivo, si se reconoce que la elección vocacional-profesional es una tarea compleja, entonces se podrán emplear pensamientos y estrategias integrales, que reconozcan no solo los aspectos personales del individuo, sino también los elementos sociales, políticos, económicos y culturales que contextualizan esa elección (Nava, 1993).

\section{Conclusiones}

El estudio de las relaciones entre orientación, educación, mercado laboral y globalización constituye una oportunidad para contextualizar su análisis y comprensión desde la perspectiva económica, social y cultural del nuevo milenio. Por ello, las políticas, necesidades y requerimientos académico-laborales, así como las estrategias y acciones que deben asumir las instituciones educativas - y sobre todo sus actores - se deben estudiar para afrontar y transformar las condiciones a las que se enfrentarán los futuros profesionales de un mundo laboral cada vez más complejo y cambiante.

Por otra parte, y como se ha planteado a lo largo de estas páginas, existen distintas posturas teórico-metodológicas que intentan explicar y comprender las relaciones entre orientación, educación, mercado laboral y globalización, las cuales van desde una visión apologético, funcional y "necesaria" para afrontar la crisis global del capitalismo hasta aquellas que la cuestionan y establecen la búsqueda de otras posibilidades que ofrezcan beneficios para la mayor parte de la población.

Por ello, en el presente ensayo se han intentado realizar aportes innovadores para el análisis no solo de los elementos teórico-empíricos y de gestión de los programas educativos 


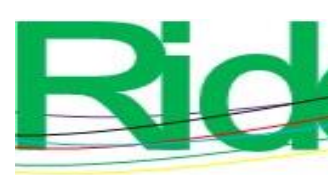

Revista Iberoamericana para la Investigación y el Desarrollo Educativo ISSN $2007-7467$

de nivel superior —específicamente de la UAEM-, sino también de las acciones de orientación en el nivel medio superior. De hecho, a partir de estas interpretaciones se pueden establecer estrategias que permitan afrontar los retos antes mencionados.

Sin duda, la preocupación del estudiante de nivel medio superior por conocer sus posibilidades reales de empleo e ingresos económicos a partir del acceso a una carrera profesional se convierte en el eje cardinal de la orientación educativa, puesto que sus expectativas de elección vocacional están encaminadas a continuar estudios de nivel superior - sobre todo con carácter propedéutico - bajo la premisa de obtener un empleo bien remunerado, lo cual reproduce los principios del capital humano (Gutiérrez, 2015).

Sin embargo, se debe pensar que la elección de una carrera trasciende el beneficio económico, pues también se deben considerar otras variables (personales, familiares, culturales, sociales e incluso políticas) determinantes para la construcción de un proyecto profesional, personal y social (Casares y Siliceo, 1996; Frankl, 2003). Por eso, desde la OE resulta necesario generar un discurso que permita vislumbrar otras posibilidades teóricometodológicas para encarar las discordancias entre orientación, mercado de trabajo y educación superior (Rascovan, 2014). Así, se puede fortalecer la formación de los orientadores desde una perspectiva que supere el enfoque "actuarial” (Bohoslavsky, 2000) que se basa en la aplicación mecánica de baterías psicométricas e información profesiográfica (a través de carteles, folletos, catálogos de carreras, etc.) que no promueven la reflexión. 


\section{Referencias}

Alles, M. (2005). Mi carrera. Cómo analizar, revisar y corregir su carrera laboral._México: Granica.

Argudín, Y. (2015). Educación basada en competencias. Recuperado de http://www.quadernsdigitals.net/index.php?accionMenu=hemeroteca.DescargaArticu loIU.descarga\&tipo=PDF\&articulo_id=7587.

Bilbao, T. (1986). Los modelos de orientación vocacional dominantes en México. México: Universidad Iberoamericana.

Bisquerra, R. (coord.) (1998). Modelos de orientación e intervención psicopedagógica. España: Praxis.

Bohoslavsky, R. (2000). Lo vocacional, teoría, técnica e ideología. Buenos Aires: Búsqueda.

Casares, D. y Siliceo, A. (1996). Planeación de vida y carrera: vitalidad personal y organizacional, desarrollo humano y crisis de madurez, asertividad y administración del tiempo. México: Limusa.

Castañeda, L. (2014). Un plan de vida para jóvenes. México: Nueva Imagen.

Castells, M., Flecha, J., Freire, P., Giroux, H., Macedo, D. y Willis, P. (1994). Nuevas perspectivas críticas en educación. España. Paidós.

Carnoy, M. (1988). La educación como imperialismo cultural. México: Siglo XXI.

Cortada, N. (1980). El profesor y la orientación vocacional. México: Trillas.

Dettmer, J. (2004). Globalización, convergencia y diferenciación de la educación superior, una revisión conceptual. Revista de la Educación Superior, 32(132).

Didriksson, A. (1997). La universidad en la transferencia de conocimientos hacia la sociedad. Políticas públicas y educación superior. México: ANUIES.

Escotet, M. (2002). Universidad y devenir, entre la certeza y la incertidumbre. Argentina: Lugar Editorial.

Frankl, V. (2003). El hombre en busca de sentido. Barcelona: Herder.

Gandini, L. (2004). La exclusión laboral juvenil en Argentina. Propuesta de una tipología para su análisis. Revista Papeles de Población, (42), 153-198.

Gómez, V. (1982). Relaciones entre educación y estructura económica: dos grandes marcos de interpretación. Revista de la Educación Superior, 11(41), 5-43.

Gutiérrez, G. (1999). La oferta y la demanda de los egresados de la UAEM: hacia otra perspectiva de la elección vocacional. Tiempo de Educar. Revista Interinstitucional de Investigación Educativa, (2), 31-69. 


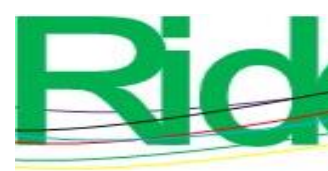

Revista Iberoamericana para la Investigación y el Desarrollo Educativo ISSN $2007-7467$

Gutiérrez, G. (2015). Análisis evaluativo del impacto del servicio de orientación educativa de nivel medio superior hacia la matrícula de licenciatura de la Universidad Autónoma del Estado de México. Toluca, México: UAEM.

Hernández Laos, E. (coord.) (2012). Mercado laboral de profesionistas en México: diagnóstico 2000-2009 y prospectiva 2010-2020: informe final. México: ANUIES.

Magaña, H. (1992). Lo que todo orientador educativo debe conocer sobre la situación actual y el futuro de las profesiones en México. Cuarto Nivel. Toluca, México: Escuela Normal Superior del Estado de México.

Márquez, A. (2011). La relación entre educación superior y mercado de trabajo en México. Una breve contextualización. Perfiles Educativos, 33, 169-185.

Mendoza, Javier. (1997). “Evaluación, acreditación, certificación: instituciones y mecanismos de cooperación”. Mungaray y Valenti. Políticas públicas y educación superior. México. ANUIES.

Mira, E. (1980). Manual de orientación profesional. Buenos Aires: Kapelusz.

Muñoz, B. y Hernández Garibay, J. (1992). Problemas y perspectivas del mercado de trabajo profesional a luz del TLC: el contexto histórico y el impacto a la orientación educativa. Cuarto Nivel. Toluca, México: Escuela Normal Superior del Estado de México.

Muñoz Varela, L. (2012). Enfoque por competencias y mercado de trabajo. Nuevas tendencias para la educación universitaria. Revista Electrónica. Actualidades Investigativas en Educación, 12(2), 1-30.

Nava, O. y Muñoz, B. (1990). La orientación educativa en México: una propuesta integradora. Memorias del II Encuentro Latinoamericano de Asociaciones y Profesionales de la Orientación. México. Universidad Autónoma de Colima.

Nava, O. (1993). La orientación educativa en México: documento base. México: Asociación Mexicana de Profesionales de la Orientación, A. C.

Navarro, J. (2014). La inserción laboral de los egresados universitarios: perspectivas teóricas y tendencias internacionales en la investigación. México: ANUIES.

Osipow, S. (1996). Teorías sobre la elección de carreras. México: Trillas.

Pescador, J. (1985). Teoría del capital humano: exposición y crítica. México: Mimeo.

Planas, J. (2003). Educación y mercado de trabajo en la globalización. Sociología de la educación. Madrid: Pearson. 

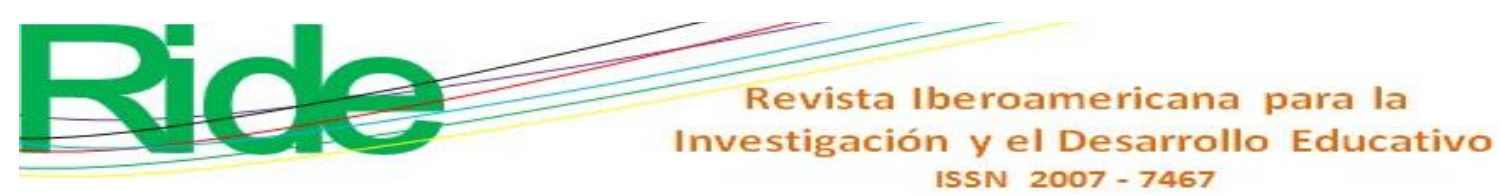

Prawda, J. (2002). Teoría y praxis de la planeación educativa en México. México: Grijalbo.

Rascovan, S. (2014). Orientación vocacional, una perspectiva crítica. México: Paidós.

Ruiz del Castillo, A. (2001). Educación superior y globalización, educar ¿Para qué? México: Plaza y Valdez.

Subsecretaría de Educación Media Superior de la SEP (2008). Reforma Integral de la Educación Media Superior. México: SEP.

Tovar, E. (2011). Plan de vida y carrera. México: Trillas.

Tobón, S. (2006). Aspectos básicos de la formación basada en competencias. Talca. Proyecto Mesesup.

Valenti, G. y Castillo, G. del (1997). Interés público y educación superior: un enfoque de política pública. Políticas públicas y educación superior. México: ANUIES. 